\title{
Technology use during pregnancy: a review of the quality of nutrition information provided by mobile phone applications.
}

\section{Abstract}

There are currently 318,000 health mobile applications (apps) available via the major app stores, with new apps appearing at a rate of approximately 200 per day. Pregnancy represents the largest sub-type within the health-related app market, providing a key route via which women who are pregnant or planning a pregnancy can obtain health information, and a cost and time efficient alternative to traditional face-to-face healthcare interactions. However the content of these applications, and therefore their ability to facilitate appropriate and safe behaviour change, is largely unmonitored and unregulated. This study aimed to objectively review the availability, affiliation and nutritional content of pregnancy apps, and specifically their adherence to current NICE guidance for optimal nutrition during pregnancy (promotion of Vitamin D and folic acid supplementation, promotion of breastfeeding). Apps $(\mathrm{n}=68)$ were retrieved from Googleplay and the Appstore using a keyword search. After exclusions ( $\mathrm{n}=39$, non-English, focused on pre- or postpregnancy, not free to access, non-functional), 29 apps were evaluated using the previously developed Mobile Application Rating Scale (MARS). The original tool was modified to include additional sub-scales to assess the nutritional content of the apps and to rate their privacy settings. A higher score for each sub scale and for the MARS score overall represents a higher quality application. Median nutrition sub-scale score (out of 5) for all apps was $3(25 \%: 2,75 \%: 4.5)$ with folic acid requirements most likely to be covered and breastfeeding least. Over one third of the apps reviewed (38\%) achieved a nutrition score $\geq 4$ and $14 \%$ ( $n=4)$ scored 5 out of 5. Nutrition score was significantly associated with overall MARS score $(r=0.615, p<0.01)$. Total MARS score (out of 5; median $3(3,4)$ ) was not associated with platform (android or apple), app rating or affiliation (e.g. Commercial, Government, NGO or academic institution), though sample size was reduced for these secondary variables. Free to access English language mobile phone applications targeted at pregnant women provide a variable quantity and quality of nutrition information. Those adhering more closely to current NICE guidelines for optimal diet in pregnancy are also more likely to be rated higher across other quality domains, however user rating does not predict information quality. This work will be used to direct interventions to improve e-health literacy amongst vulnerable groups such as pregnant women and to design more appropriate applications which are able to more effectively combine usability and acceptability with a robust evidence base.

\section{Conflict of Interest}

There is no conflict of interest 